Eine digitale Prosopographie der Führungsschichten des kaiserzeitlichen Imperium Romanum (Senatorenstand - ordo decurionum): Ihre strukturellen Notwendigkeiten

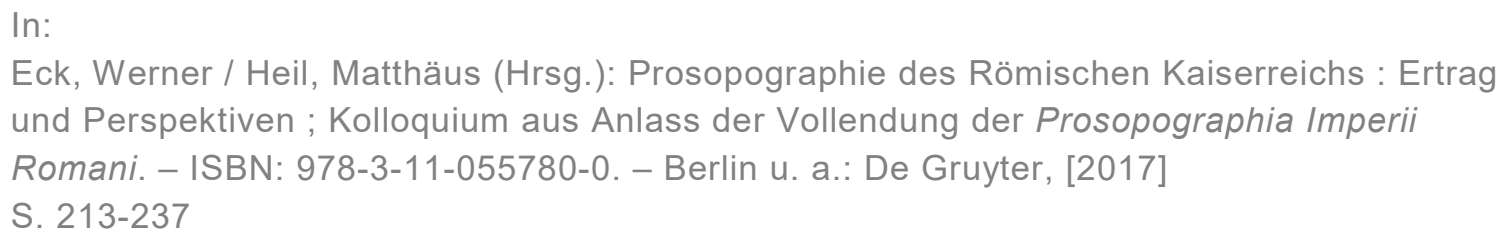




\section{Matthäus Heil \\ Eine digitale Prosopographie der Führungsschichten des kaiserzeitlichen Imperium Romanum (Senatorenstand - ordo decurionum): Ihre strukturellen Notwendigkeiten}

Es kann missverstanden werden, wenn Mitarbeiter eines abgeschlossenen Projekts über dessen Fortsetzung oder Neuauflage nachdenken. Denn allzu nahe liegt der Einwand, hier wolle sich eine Institution doch nur perpetuieren - womit unterstellt wird, es gehe in Wahrheit um partikulare Interessen und alles andere sei bloß vorgeschoben. Im Falle der Prosopographia Imperii Romani (PIR) ist dieses beliebte Universal-Argument aber nicht am Platz. Die PIR ist definitiv abgeschlossen, es sind keine übriggebliebenen Angestellten mehr zu versorgen und die ehemals Beteiligten sind in ihren Forschungsinteressen auch nicht ausschließlich auf die Prosopographie fixiert. So geht es bei den folgenden Überlegungen tatsächlich nur darum, ob eine neue PIR sinnvoll wäre und wie sie dann beschaffen sein müsste unabhängig von der Frage, wer gegebenenfalls an welchem Ort mit welcher Finanzierung daran arbeiten würde. Der Beitrag steht daher unter dem Vorbehalt, dass eine Grundsatzentscheidung noch nicht gefallen ist; er steht sozusagen vom Anfang bis zum Ende im Konjunktiv.

\section{Prosopographie zwischen Buch und Internet}

Was folgt, ist aber nicht einfach gelehrter Muße entsprungen, sondern steht in einem elementaren Zusammenhang mit der soeben abgeschlossenen zweiten Auflage der PIR und ist sozusagen aus ihr hervorgegangen. Das Projekt lief über sehr lange Zeit. ${ }^{1}$ Als die letzte Generation der Bearbeiter die Aufgabe von ihren Vorgängern übernahm, sah sie sich mit Grundsatzfragen konfrontiert, ohne sie frei beantworten zu können, nämlich: Ist das Projekt

1 Zur Geschichte des Unternehmens siehe den Beitrag von Werner Eck in diesem Band. 
überhaupt noch sinnvoll? Und: Würde man tatsächlich alles genauso machen, wenn man von den ererbten Vorgaben frei wäre? Die Antwort war zunächst einmal pragmatischer Art: Ein Neuansatz kurz vor Schluss hätte dazu geführt, dass das schon Vorliegende unvollendet liegengeblieben wäre, ohne dass stattdessen etwas Besseres hätte zum Abschluss gebracht werden können. Um nicht ein Trümmerfeld zu hinterlassen, blieb nur, die PIR im Wesentlichen so zu Ende zu führen, wie sie begonnen worden war, und das tradierte Muster allenfalls in Details abzuändern. Auch die lateinische Sprache wurde beibehalten, doch wurde ein möglichst klares und verständliches Latein angestrebt. So weit wie möglich wurden die Arbeitsmittel der Gegenwart benutzt: Die Namensliste - das Rückgrat des Lexikons - wurde elektronisch geführt und seit Mitte der 1990er Jahre auch die Materialsammlung; die Druckvorlagen wurden am Computer erstellt. Die Veröffentlichung von Nachträgen im Internet musste allerdings zugunsten der Buchpublikation wieder einstellt werden, obwohl diese ein positives Echo gefunden hatte. Nur durch diese Selbstbeschränkung war es zu erreichen, dass heute ein fertiges Werk vorliegt.

Aber die Grundfrage ist damit eigentlich nicht beantwortet; sie wurde nur etwas verschoben. Genauer genommen stellt sie sich auf mehreren Ebenen: einer prinzipiellen, einer konzeptionellen und einer der technisch-organisatorischen. Ob es überhaupt sinnvoll ist, Prosopographie zu betreiben, braucht man wohl nicht in extenso zu diskutieren. Die teils ideologisch aufgeladenen Grundsatzdebatten sind eher eine Sache der Vergangenheit, ${ }^{2}$ und der personenkundliche Ansatz bietet nach wie vor wesentliche Erkenntnismöglichkeiten. ${ }^{3}$ Und zumindest als praktische Arbeitsmittel haben sich pro-

2 Siehe dazu kurz M. Heil: Friedrich Münzer und die prosopographische Methode - Rückblick und Ausblick, in: M. Haake - A.-C. Harders (Hgg.): Politische Kultur und soziale Struktur der Römischen Republik. Bilanzen und Perspektiven. Akten der internationalen Tagung anlässlich des 70. Todestages von Friedrich Münzer (Münster, 18.-20. Oktober 2012), Stuttgart 2017, 91-110. Die Kritiker eines prosopographischen Zugriffs sehen häufig Ideen oder andere Abstrakta als die Triebfeder der Geschichte oder sind einem Konzept von Sozialgeschichte verpflichtet, in dem nur anonyme Gruppen vorkommen, aber keine als Individuen erkennbaren Menschen.

3 Siehe den Beitrag von Marietta Horster in diesem Band. - T. D. Barnes, Prosopography and Roman History, in: K. S. B. Keats-Rohan (Hg.), Prosopography Approaches and Applications. A Handbook, Oxford 2007, 84 hat zurecht angemerkt, dass auch diejenigen, die auf einer theoretischen Ebene die sprosopographische Methoder kritisieren, in praxi selbst auf prosopographische Erkenntnisse zurückgreifen. 
sopographische Nachschlagewerke bestens bewährt. Denn es versteht sich, dass man alle Hintergrundinformationen auch zu den weniger prominenten historischen Gestalten prüfen sollte; doch wäre der Arbeitsaufwand zu groß, wenn jeder für sich selbst die nötigen Daten zusammentragen müsste. So ist es geradezu ein Gebot der ökonomischen Vernunft, dass die Informationen an einer Stelle gesammelt und allen Interessierten zur Verfügung gestellt werden.

Wesentlich mehr des Nachdenkens wert scheint die Frage, wie eine neue PIR idealerweise beschaffen sein müsste. Das Wichtigste liegt auf der Hand: Die kaiserzeitliche Prosopographie kennt keine "stabilitas loci«. Denn ständig kommen neue Quellen hinzu, und damit wandelt sich auch ständig die Diskussionsgrundlage. Bislang kamen die prosopographischen Standardwerke mit dieser an sich faszinierenden Herausforderung nicht gut zurecht. Auch die eben abgeschlossene PIR bot als gedrucktes Lexikon nur den Kenntnisstand der jeweiligen Abfassungszeit. Man hat versucht, sich mit Addenda zu behelfen - das taten bereits die Bearbeiter der ersten Bände und das ist auch in den allerletzten Bänden geschehen. Mehr als eine Notlösung war dies aber nicht, und die frühen Bände der zweiten Auflage der PIR sind inzwischen an vielen Stellen sehr veraltet.

Mittlerweile gibt es ein Alternative zum gedruckten Lexikon: Datenbanksysteme und elektronische Publikationen. Damit ließe sich das alte Erbübel tatsächlich beheben; die Dokumentation könnte auf dem Stand des aktuellen Wissens gehalten werden. Daher würde eine neue PIR digital sein oder sie würde gar nicht sein. Selbstverständlich sollte sie über das Internet zugänglich sein und open access bieten, also für alle Interessenten kostenfrei zur Verfügung stehen. ${ }^{4} \mathrm{Ob}$ man für die Liebhaber des gedruckten Buches irgendwann quasi eine Momentaufnahme auch einmal ausdruckt, könnte man später überlegen. Möglich wäre es, nötig aber nicht.

4 Von den Verfechtern des open access wird oft an den Rand geschoben, dass auch in diesem Publikationsmodell Kosten (von teils beträchtlicher Höhe) anfallen und dass eine der zentralen Fragen darin liegt, wie diese bestritten werden sollen. Wenn für den Endbenutzer keine Kosten entstehen sollen (außer für gelegentliche einzelne Computer-Ausdrucke), bleibt eigentlich nur, dass die anbietende Institution oder der Drittmittelgeber die finanziellen Lasten übernimmt. Denn wenn die Forscher selbst dafür zahlen müssten, um überhaupt publizieren zu dürfen, würden sich für sie die Publikationsmöglichkeiten durch open access in den meisten Fällen deutlich verschlechtern. Eine weitere offene Frage hängt ebenfalls primär an der Finanzierung, nämlich wie sichergestellt werden kann, dass elektronische open access-Publikation dauerhaft (d.h. über viele Jahrzehnte) verfügbar bleiben. 
Eine Internet-Publikation böte überdies die Chance, eine direkte Beziehung zu anderen Internet-Projekten herzustellen. Die PIR ist auf Zuarbeit angewiesen, auf gute, verlässliche Quellenpublikationen, und ferner auf die im Lauf der Zeit kontinuierlich erscheinende Fachliteratur. Statt all dies nur zu zitieren, eröffnet sich nun die Möglichkeit einer unmittelbaren Zusammenschau. Wenn in der elektronischen PIR auf ein Quellenzeugnis Bezug genommen wird, kann der Benutzer unmittelbar zu dessen digitaler Edition springen (sofern vorhanden) und sich sogleich ein eigenes Urteil bilden und dann zur PIR zurückkehren. In solchen Editionen werden heute meist auch Bilder des Monuments angeboten, denen nicht selten Informationen zu entnehmen sind, die weit über den blanken Inschriftentext hinausgehen. ${ }^{5}$ Umgekehrt kann eine digitale Inschriften-Edition den Leser für weitergehende Informationen einfach zur PIR weiterleiten, wenn es um eine prominente Persönlichkeit der Kaiserzeit geht, und damit auch ihren eigenen Kommentar wirkungsvoll entlasten. Beide Werke lassen sich auf diese Weise effizienter und zugleich kritischer benutzen. Überdies können beide Seiten auf Referate, was an der jeweils anderen Stelle steht, weitgehend verzichten und sich mehr auf ihre Kernaufgaben konzentrieren. Hingegen würde es sicher niemand verstehen, wenn ein Internet-Projekt autark und isoliert existieren wollte, denn dies widerspräche dem Prinzip des Mediums. Technisch sind die skizzierten Verknüpfungen bereits möglich und einige Versuche der Umsetzung wurde auch bereits unternommen: Die digitale Edition der spätantiken Inschriften von Aphrodisias ${ }^{6}$ verwies auf die digitalen Addenda der PIR, wo es angebracht war. Und 2011 wurde zusammen mit Charlotte Roueché und Gabriel Bodard ein britisch-deutsches Projekt einer digitalen Kern-PIR im Verbund mit Inschrifteneditionen vorbereitet, das allerdings wegen fehlender Finanzierung nicht zur Ausführung kam.

Die Idee einer digitalen Prosopographie entstammt also sachimmanenten Überlegungen und reagiert auf Probleme der überkommenen Arbeitsweise. Sie ist nicht ein Anliegen von Hobby-Informatikern unter den Altertumswissenschaftlern, denen es um technisches Experimentieren geht, und sie ist auch nicht dem derzeitigen Boom der "digital humanities" geschuldet, wo das Pferd oft vom Schwanz her aufgezäumt wird. Denn man hat nicht selten den Eindruck, dort würden technische Lösungen angeboten, die noch

5 Dazu in Kürze W. Eck: Tradition and Progress. The Roman World seen through Inscriptions in the Digital Age (im Druck).

6 http://insaph.kcl.ac.uk/ala2004/. 
auf der Suche nach einem geeigneten Problem sind, und es scheint so, als würde sich die Technik auf Kosten der Inhalte verselbständigen.

Allerdings gibt es inzwischen eine Reihe von Erfahrungen mit Computerprojekten in den Geisteswissenschaften - auch solche der eher traurigen Art. Viele Projekte wurden groß angekündigt und starben dann eines stillen Todes (oder liegen als hoffnungsloser Fall auf der Intensivstation). Eigentlich kennt man auch die wichtigsten Gründe. Schlecht ist es, sich zunächst für eine bestimmte Software zu entscheiden und erst dann zu überlegen, was genau man tun will. Und oft wurde erst während der laufenden Arbeit bemerkt, dass der gewählte Ansatz in kaum lösbare Probleme führt. Manchmal hat man wohl auch entsprechende Warnungen nicht verstanden, die allerdings für einen Projektverantwortlichen ohne tiefere Sachkenntnis oft nur schwer von bloßer Bedenkenträgerei zu unterscheiden sind. Bei Datenbanken, die in der Regel den Kern solcher Projekte bilden, werden die entscheidenden Fehler in aller Regel bereits gemacht, bevor der erste Datensatz eingegeben ist, nämlich bei den grundlegenden Strukturentscheidungen. Wenn man Erfolg haben will, benötigt man präzise beschriebene Ziele und klare, eindeutige Begriffe - und man sollte im Voraus an zukünftige Eventualitäten denken. In mancher Hinsicht ist der Computer auch eine Wahrheitsmaschine, die gnadenlos aufzeigt, ob Konzeptionen tragfähig sind oder nicht.

So ist mit einem Plädoyer für eine digitale Prosopographie noch nicht viel gesagt. Es bleibt eine Sonntagsrede, solange man nicht auch aufzeigt, wie sie auszugestalten wäre und wie sie realistischerweise verwirklicht werden könnte.

\section{Nachträge oder Neubeginn?}

Die erste Grundfrage ergibt sich bereits aus dem Gesagten: Wie sollte sich eine digitale PIR zu ihrer gedruckten Vorgängerin verhalten? Sollte sie das Supplement zu PIR ${ }^{2}$ sein, das immer wieder aktualisiert wird? Oder sollte man einen Schlussstrich ziehen und noch einmal neu anfangen?

Schon Edmund Groag und Arthur Stein haben klar gesehen, dass umfangreiche Supplemente meist unübersichtlich und schwer zu handhaben sind, und deswegen haben sie schließlich die Berliner Akademie dazu gebracht, statt der seit 1897 geplanten Addenda zu PIR ${ }^{1}$ eine zweite Auflage zu erarbeiten. Bei gedruckten Büchern würden früher oder später Supplemente zu den Supplementen nötig, womit die Übersicht wohl endgültig verloren 
ginge. Digitale Addenda hätten wenigstens dieses letzte Problem nicht; man könnte sie immer wieder auf den aktuellen Stand bringen. Aber man würde ein Hybrid produzieren, denn der Benutzer müsste jeweils zugleich das Buch vor sich liegen haben und am angeschalteten Computer sitzen. Vor allem aber bleiben alle Supplemente - gleich welcher Art - an die Struktur des Stammwerkes gebunden. Nur wenn diese nicht angetastet werden muss, sind sie das Mittel der Wahl. Aber genau dies steht bei der PIR in Frage. Nicht selten führen Neufunde dazu, dass alte Artikel eigentlich komplett umgeschrieben werden müssten. So hat sich bei einem M. Calpurnius Rufus erwiesen, dass die im Artikel zusammengezogenen Daten in Wahrheit zu drei verschiedenen Personen gehören. ${ }^{7}$ Umgekehrt müssten oft zwei Artikel zu einem einzigen vereinigt werden. Und selbstverständlich kommen immer neue Personen hinzu, was auch Auswirkungen auf alte Artikel hat. Auch das Hybrid würde also rasch unübersichtlich und schwer handhabbar werden. Für grundlegend neue Ideen wäre ohnehin kein Raum.

Die hauptsächlichen Vorteile einer digitalen PIR kämen also nur dann zur Geltung, wenn man sie von alten Erblasten befreit. So erscheint ein harter Schnitt, ein Neuanfang als die wohl einzige sinnvolle Lösung.

Allerdings wäre dies zunächst der aufwendigere Weg. Immerhin könnte man die Namensliste und die digitale Materialsammlung weiterverwenden und daraus die Basis für etwas Neues formen; die Daten müssten allerdings tiefgreifend umstrukturiert werden. Für die anderen digitalen Hinterlassenschaften - insbesondere die Druckdateien ab dem Buchstaben $\mathrm{P}$ - gäbe es keine Verwendung mehr; sie müssten archiviert werden.

\section{Elitenprosopographie}

Wenn man einen Neuanfang wagt, kann man frei und neu darüber nachdenken, welcher Konzeption von Prosopographie man folgen soll. Die PIR galt als das Musterbeispiel von `Elitenprosopographie;; sie ist freundlich gesagt das 'Who is Whor des römischen Kaiserreichs oder etwas weniger freundlich formuliert: weitgehend das Verzeichnis der Reichen und Mächtigen. Sollte man sich weiter darauf beschränken? Anders als beim traditionellen Lexikon in Buchform fiele bei einer digitalen Prosopographie das Argument des beschränkten Platzes weg; heute sind große Mengen Speicherplatz leicht und kostengünstig verfügbar.

7 M. Calpurnius Rufus, PIR ${ }^{2}$ C 313; vgl. PIR ${ }^{2}$ VII 1 p. 117 sq. 
Dennoch würde man sich wohl keinen Gefallen tun, würde man alle Schranken fallen lassen. Dagegen sprechen sowohl die Natur der Quellen als auch inhaltliche Gründe. Was würde man gewinnen, wenn man z. B. alle bezeugten Personen der Kaiserzeit aufnähme? Solche Versuche gibt es, aber der Sinn hat sich noch nicht erschlossen. Denn von den allermeisten gewöhnlichen Leuten kennen wir nur ihren Grabstein und erfahren lediglich, dass sie existiert haben; vielleicht können wir noch den Namen des Ehepartners notieren - aber selten viel mehr. Im Allgemeinen kann man nur von den Angehörigen der höheren Stände so viel in Erfahrung bringen, dass sie wenigstens in Umrissen als Personen erfassbar werden. Denn die Wahrscheinlichkeit der Erwähnung in den Quellen steigt in aller Regel mit dem sozialen Status. Prosopographien zu `kleinen Leuten ‘ lohnen sich zumeist nur, wenn sie auf eine klar umrissene Gruppe fokussiert und mit einer sinnvollen Fragestellung verbunden sind. ${ }^{8}$

Ferner wurden in der Kaiserzeit die Statusunterschiede in einer - zumindest für heutiges, zentraleuropäisches Empfinden - geradezu provozierenden Offenheit und Deutlichkeit zur Schau gestellt und Standesüberschreitungen wurden in aller Regel als ein Übel gebrandmarkt. Vereinfacht gesagt, herrschte das Prinzip der Ungleichheit. Dem werden die Forschung und ihre Instrumente Rechnung tragen müssen. Man würde sozusagen den Gegenstand verfehlen, wenn man die offen zelebrierte Ungleichheit mit einem Forschungsansatz anginge, der die allgemeine Gleichheit zur Grundidee hat. ${ }^{9}$ Die Beschränkung auf die Elite war und ist eine aus der Sache sich ergebende sinnvolle Entscheidung.

8 Siehe beispielsweise H. Leppin: Histrionen. Untersuchungen zur sozialen Stellung von Bühnenkünstlern im Westen des Römischen Reiches zur Zeit der Republik und des Principats (Antiquitas R. 1, 41), Bonn, 1992.

9 Vgl. J. Rüpke: Fasti Sacerdotum. Die Mitglieder der Priesterschaften und das sakrale Funktionspersonal römischer, griechischer, orientalischer und jüdischchristlicher Kulte in der Stadt Rom von 300 v. Chr. bis 499 n. Chr. (Potsdamer altertumswiss. Beiträge 12,1-3), Stuttgart 2005. Nüchtern besehen, zeigt sich hierbei, dass das `Funktionspersonal keine einheitliche Gruppe war, sondern regelrecht in die staatlichen Priester einerseits und die Priester je spezifischer, privater Kultvereinigungen andererseits zerfiel - nur erstere gehören jeweils zur Spitze der Gesellschaft (und fanden Aufnahme in die PIR). 


\section{Reichsaristokratie und lokale Eliten}

Damit aber stellt sich die Frage: Wer gehört zur Elite? Oder anders: Ist die zeitliche und inhaltliche Abgrenzung der bisherigen PIR wirklich überzeugend? An der Beschränkung auf die römische Kaiserzeit (von 30 v. Chr. bis 284 n. Chr.) gibt es im Grundsatz wohl wenig zu diskutieren. Für diese Epoche gibt es zudem eine spezifische Quellenlage mit einer herausragenden Bedeutung von Inschriften, die für andere Zeiten nicht besteht. Allenfalls könnte man erwägen, ob man die Ära Diocletians noch ganz dazu nimmt.

Erheblich weniger überzeugend ist die Definition des Kreises der aufzunehmenden Personen. Mommsen hatte ihn am Anfang der ersten Auflage der PIR etwas freihändig festgelegt: Es ging ihm darum, die snamhaften Männer (und Frauen) der Epoche zu erfassen, also die Führungsschicht des Reiches, und dabei sollte der Befund in den Inschriften mit dem Bestand der literarisch überlieferten Quellen in Verbindung gebracht werden. Erfasst werde sollten hierzu die Senatoren und die Ritter höheren Ranges mitsamt ihren Familien, natürlich die Kaiser und ihr Haus, ferner die mit Rom verbundenen auswärtigen Herrscher und schließlich alle Personen, die in der Literatur vorkommen (mit Ausnahme der Christen).

Es versteht sich, dass man wenigstens eine grobe Abgrenzung des Personenkreises benötigte, als man mit der Materialsammlung begann. Aber es ist dann auf Dauer bei dieser Arbeitsdefinition geblieben. Wie Werner Eck dargelegt hat, ${ }^{10}$ entwickelte sich die zweite Auflage der PIR aus geplanten Nachträgen für die erste, womit sie auch die Festlegungen aus der ersten Auflage quasi ererbt hat. Daran musste bis zum Ende festgehalten werden. Mit einem Neuanfang gäbe es nun die Gelegenheit einer Evaluation im Lichte der inzwischen gewonnenen Erkenntnisse.

Es springt ins Auge, dass in Mommsens Arbeitsdefinition Kriterien unterschiedlicher Art amalgamiert wurden: die Zugehörigkeit zu einem Stand und die Erwähnung in einer Quellengruppe. Das führt mitunter zu etwas seltsamen Einträgen in der PIR. Aufgenommen werden musste beispielsweise Thestylus (PIR $\left.{ }^{2} \mathrm{~T} 185\right)$, der hübsche Sklavenjunge eines Voconius Victor sowie dieser Voconius Victor selbst $\left(\mathrm{PIR}^{2}\right.$ V 992), weil beide bei Martial vorkommen. ${ }^{11}$ Aber weder der Sklavenjunge noch sein Besitzer hatten eine größere Bedeutung - und es muss Tausende von Lustknaben geben haben und Tausende von lüsternen Sklavenbesitzern, die unberücksichtigt blie-

10 Siehe seinen Beitrag in diesem Band.

11 Mart. 7,29; 8, 63. 
ben. Auch Vettius Valens wurde aufgenommen $\left(\mathrm{PIR}^{2} \mathrm{~V}\right.$ 493), weil er sich selbst in seinem noch erhaltenen Handbuch zur Astrologie erwähnt. Doch war er offenkundig nicht mehr als ein kleiner Geschäftemacher, der irgendwo in Ägypten seine Dienste anbot. Für viele Ärzte gilt ähnliches. Das Kriterium `Person in der Literatur` wird man also fallenlassen müssen.

Auch unter den auswärtigen Königen kommt verschiedenes zusammen. Der Kriterien wegen wurden auch der Partherkönig Vologaeses I. (PIR ${ }^{2} \mathrm{~V}$ 940) und der Sasanide Šapur (PIR ${ }^{2}$ S 182) aufgenommen und - hoffentlich - angemessen behandelt. Aber man kann sich fragen, was diese großen Gegner Roms in einer römischen Prosopogaphie zu tun haben. Besser sollte man sich auf die Könige beschränken, die Teil des römischen Herrschaftsverbands waren. Dass ein König des bosporanischen Reiches auf der Krim bei sich zu Hause eine Statue mit einer Inschrift für einen Kaiser aufstellte und

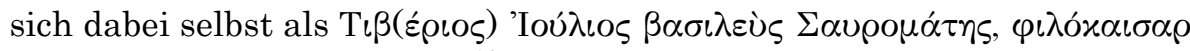

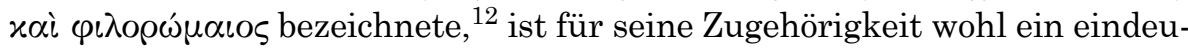
tiges Indiz. Bei Šapur findet man natürlich nichts dergleichen.

Bei den Kaisern wurde nie eine vollständige Dokumentation angestrebt (und dennoch gehören die PIR-Artikel bei manchen Kaisern zur grundlegenden Literatur). Vollständigkeit wird da wohl auch in Zukunft niemand erwarten. ${ }^{13}$

Den eigentlichen Kern stellte die Dokumentation über den Personenkreis dar, den man grob als die Reichselite bezeichnen könnte. Deren Kenntnis bildet ein wichtiges Korrektiv zur Historiographie der Kaiserzeit, die bekanntlich auf die Person des Kaisers fixiert ist, obwohl es nur bedingt auf dessen persönliche Leistung ankam. Die Herrschaftsausübung wurde jedenfalls zum großen Teil von den hohen Funktionsträgern wahrgenommen. Auch deren Verwandte aufzunehmen, war richtig, denn die Familien waren untereinander stark verflochten. Aber wo kann man sinnvollerweise eine Grenze zu ziehen? Mittlerweile hat sich in umfangreichen Forschungen gezeigt, dass es in sozialer Hinsicht zwischen den Inhabern der hohen Reichsämter und den lokalen Honoratioren keine festen Schranken gab. In der frühen Kaiserzeit stiegen Leute aus der Honoratiorenschaft italischer Städte in den Senat auf, später solche aus Spanien und Südgallien und dann auch solche

12 IGR I 879; vgl. PIR ${ }^{2}$ I 551.

13 Allerdings sind nicht alle Kaiser gleichmäßig gut erforscht und für manche von ihnen sind die PIR-Artikel - gerade was die Basisdaten anlangt - noch immer zu konsultieren. Vgl. D. Kienast - W. Eck - M. Heil: Römische Kaisertabelle. Grundzüge einer römischen Kaiserchronologie. 6., überarbeitete Auflage, Darmstadt 2017. 
aus dem griechischen Osten und aus Africa. Im frühen dritten Jahrhundert saßen Männer aus fast allen Teilen des Imperiums im Senat. ${ }^{14}$ Bei den hohen ritterlichen Ämtern galt all dies erst recht, zumal fast alle Procuratoren zuvor Dienst als Offizier geleistet hatten. Die Senatoren und ranghohen Ritter behielten in der Regel ihre Bindung an Region ihrer Herkunft bei oder ein Teil der Familie blieb dort präsent. Erst ab der Zeit der ıSoldatenkaiser löste sich der Zusammenhang zwischen den städtischen Honoratioren und den hohen Funktionsträgern des Reiches teilweise auf und in der Spätantike ist man mit ganz anderen Verhältnissen konfrontiert. ${ }^{15}$

Der Konnex zwischen Reichsbeamten und lokalen Honoratioren ist in vielen Fällen gut bezeugt, nicht nur bei den notorischen Vedii aus Ephesos. ${ }^{16}$ Beispielsweise gab es einen T. Flavius Athenagoras Agathos ( $\mathrm{PIR}^{2}$ F 223) aus Aphrodisias, Sohn eines Flavius Mithridates Agathos aus einer altansässigen und offensichtlich sehr wohlhabenden Familie (vgl. das beigefügte Stemma). Er selbst brachte es bis zum kaiserlichen Procurator und heiratete eine Sallustia Frontina ( $\mathrm{PIR}^{2}$ S 104), Tochter eines Senators und Schwester eines Senators (ihre Familie stammte vermutlich aus Italien). Aus der Ehe ging ein Sohn namens T. Sallustius Flavius Athenagoras ( $\mathrm{PIR}^{2} \mathrm{~S}$ 89) hervor, der in den Senat aufstieg, und ferner eine Tochter namens Flavia Apphia ( $\mathrm{PIR}^{2}$ F 412), die offenkundig zu Hause bleib und Archierea der Provinz Asia wurde. Diese wiederum heiratete einen M. Ulpius Carminius Claudianus (PIR ${ }^{2}$ C 433), dessen - offensichtlich ebenfalls sehr reiche - Familie aus Attouda stammte und dessen Vater Asiarch war, also zu den angesehensten Personen der Provinz gehörte. M. Ulpius Carminius Claudianus selbst hatte verschiedene hohe Ämter in der Provinz und in mehreren Städten inne. Aus der Ehe mit Flavia Apphia ging - unter anderem - ein T. Carminius Flavius Athenagoras Claudianus ( PIR $^{2}$ C $429+$ F 224) hervor, der Senator wurde und es bis zum Suffektkonsulat brachte, aber in den Bürgerkriegen ab 193 n. Chr. unter die Räder gekommen zu sein scheint. Sein Bruder, der wohl mit vollem Namen M. Ulpius Carminius Claudianus hieß (PIR ${ }^{2}$ C 431), blieb in der Provinz Asia und wurde dort Asiarch. Die Kinder des Senators T. Carminius Flavius Athenagoras Claudianus konnten sich im Senat behaupten; bezeugt sind seine Söhne M. Flavius Carminius Athenagoras Livianus

14 Siehe Atti del Colloquio Internazionale AIEGL su Epigrafia e Ordine Senatorio, Rom 1982, besonders Band 2.

15 Siehe dazu kurz M. Heil: Der Ritterstand, in: K.-P. Johne u. a. (Hgg.): Die Zeit der Soldatenkaiser. Krise und Transformation des Römischen Reiches im 3. Jahrhundert n. Chr. (235-284), Bd. 2, Berlin 2008, 737-761.

$16 \mathrm{PIR}^{2}$ V 316-318, 322, 325-327 und ebd. Stemma 7. 
Eine digitale Prosopographie

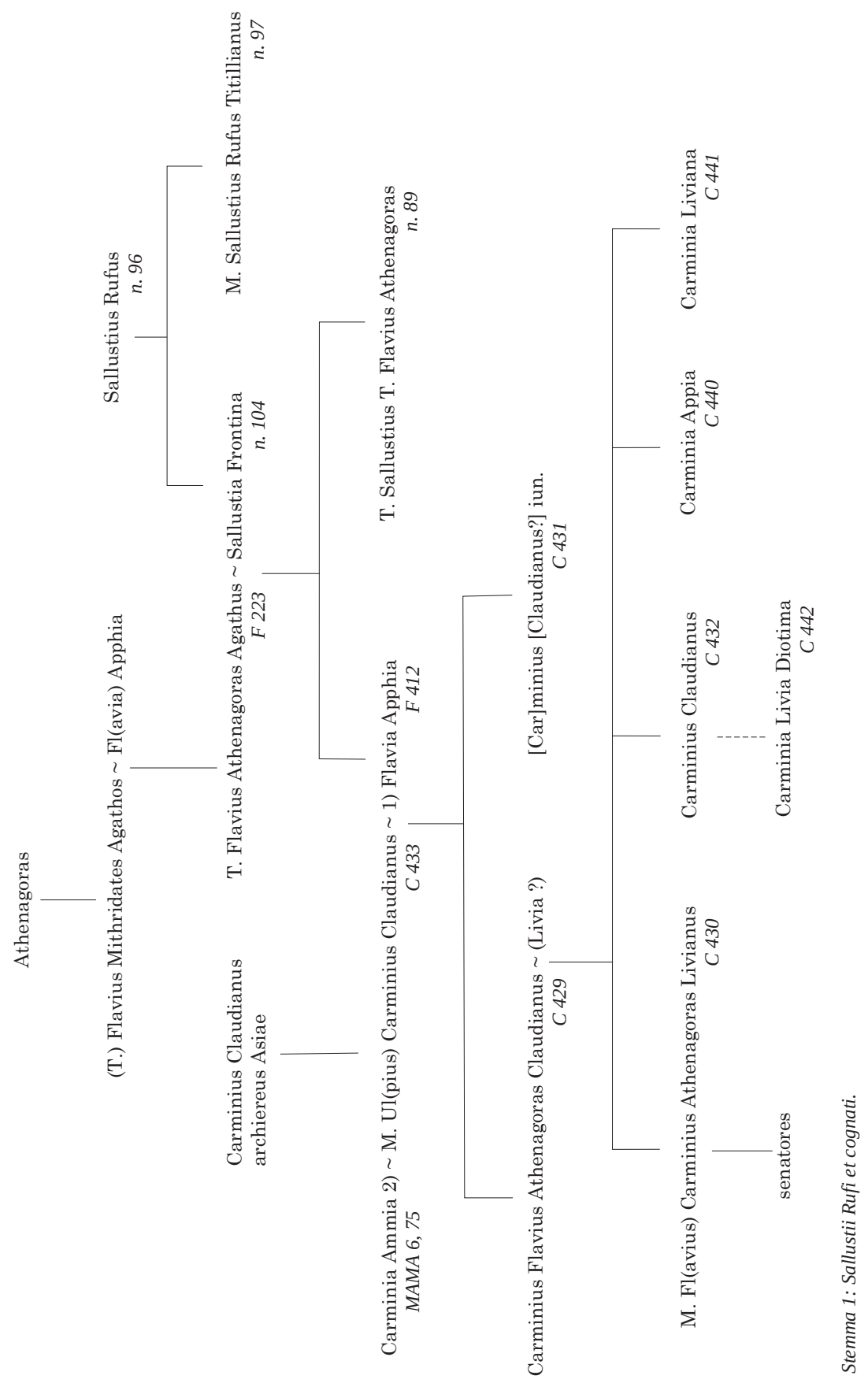


( $\mathrm{PIR}^{2} \mathrm{C} 430$ ) und Carminius Claudianus ( $\mathrm{PIR}^{2} \mathrm{C} 432$ ) sowie seine Töchter Carminia Apphia ( $\mathrm{PIR}^{2} \mathrm{C} 440$ ) und Carminia Liviana (PIR ${ }^{2} \mathrm{C} 441$ ). Die Familie in ihren Verzweigungen noch weiter zu verfolgen, wäre möglich, ist hier aber sicher nicht nötig.

Man sieht hier, wie diejenigen Familienmitglieder, die in hohe Reichsämter aufstiegen, nur einen Teil eines umfangreichen Geflechts ausmachten. Zugleich deutet sich an, dass die reichen lokalen Honoratioren für gewöhnlich nicht nach sunten heirateten, sondern sich eher mit ihresgleichen aus anderen Städten verbanden. Das bestätigt sich vielfach. Entsprechend waren Personen aus diesen Kreisen recht oft im mehreren Städten aktiv. Der bekannte Ti. Iulius Celsus Polemaeanus (PIR I 260, Suffektkonsul 92 n. Chr.) stammte eigentlich aus Sardeis, hat aber der Stadt Ephesos seine berühmte Bibliothek hinterlassen. Analoges kann man auch in Spanien und anderswo finden.

Wenn man all dies ernst nimmt - und man sollte es tun -, bleibt nur eine Konsequenz: die lokalen Honoratioren (und nicht nur die Ritter) müssten in einer neuen PIR mit aufgenommen werden. Oder anders: Der Begriff der Führungsschicht des Imperiums müsste wesentlich erweitert werden. Er würde dann alles umfassen, was als die ordines bezeichnet wurde. ${ }^{17}$ Würde man vor dieser Schlussfolgerung zurückschrecken, würde man zu keiner halbwegs in sich konsistenten Grenzziehung gelangen.

Freilich sei unumwunden zugegeben, dass dies erhebliche praktische und arbeitsorganisatorische Konsequenzen nach sich zöge: Die Zahl der zu berücksichtigenden Personen - derzeit ca. 15.000 - würde stark zunehmen, sich wahrscheinlich mehr als verdoppeln. Allerdings würden nur für wenige unter den neu Hinzukommenden sehr umfangreiche Einträge nötig. Aufgearbeitet sind diese Personen bislang nur in Teilen und das Wissen ist auf eine Vielzahl von Publikationen verstreut. Für die Ritter, die eine Offiziersstelle innehatten, kann man auf das Werk von Hubert Devijver zurückgreifen - ein inzwischen abgeschlossenes und selbst wieder ergänzungsbedürftiges Projekt. Auch die Provinzialpriester in den wichtigsten Provinzen sind erfasst. ${ }^{18}$ In Teilbereichen müsste man aber noch Pionierarbeit leisten. Umso klarer und tragfähiger würde dann der Befund, den man bekommt.

17 Weiterer Überlegungen bedarf es allerdings, wie und nach welchen Kriterien man die Angehörigen der lokalen Honoratiorenschaft eindeutig identifizieren und von anderen abgrenzen kann; siehe den Beitrag von Marietta Horster in diesem Band.

18 Siehe beispielsweise M. D. Campanile: I sacerdoti del koinòn d'Asia (I sec. a.C. - III sec. d.C.). Contributo allo studio della romanizzazione delle élites provinciali nell'Oriente greco (Studi ellenistici 7), Pisa 1994. 


\section{Was heißt >Person`?}

Bevor man sich in die Details der technischen Umsetzung stürzt, sollte man zunächst einen zweiten Blick auf die Frage werfen: Was genau ist das, was abgebildet werden soll? Dies mag pedantisch klingen, da die Antwort doch anscheinend auf der Hand liegt: Personen. Aber wie die praktische Arbeit zeigt, bleibt es oft strittig, wem welches Zeugnis zuzuweisen ist, was zu unterschiedlich rekonstruierten Biographien führen kann. Insofern ist >Person in der Prosopographie wohl doch keine so eindeutige Basisgröße, dass ein Computer gut damit arbeiten kann.

Ein Projekt der BBAW, das >Personendatenrepositorium`(PDR), das mit ähnlichen Problemen bei Personen der Neuzeit befasst ist, hat daraus eine sehr radikale Konsequenz gezogen, zumindest in der Theorie. Der Begriff der Person wird dort faktisch entkernt und man zieht sich auf die Einzelzeugnisse als Basisgröße zurück. "Eine Person wird ... als die Menge aller Aussagen definiert, die zu ihr getroffen werden. Damit ist es möglich, sowohl sich ergänzende als auch sich widersprechende Aussagen nebeneinander abzubilden, was grundlegenden Problemen biografischen Arbeitens Rechnung trägt. Dieses Konzept weicht von dem herkömmlichen Ansatz ab, Personen als Datensätze mit vordefinierten Eigenschaften zu erfassen. ${ }^{19} \mathrm{In}$ praxi kommt man dann aber doch dahin, dass Aussagen einer Person zugeordnet werden.

Hier wurde zweifellos ein Problem erkannt, aber wohl nicht zureichend gelöst - zumal grundlos unterstellt wird, alle Aussagen seien gleichwertig. Der Begriff der Person ist jedoch nicht verzichtbar und bleibt grundlegend; er ist das Axiom der ganzen Disziplin. Davon zu unterscheiden ist, was wir über eine Person wissen: Unsere Kenntnis ist oft begrenzt und defektiv. Vor dieser Einsicht kann man kapitulieren oder man kann sie als Herausforderung nehmen, Wissenschaft im Sinn eines immer weiter fortschreitenden Erkenntnisprozesses zu betreiben. Was die PIR betrifft, wird kaum ein verständiger Benutzer je angenommen haben, dass ihm dort Tatsachenaussagen - in einem philosophischen Sinn - präsentiert würden; es dürfte immer klar gewesen sein, dass man es im Zweifelsfall mit einer wissenschaftlichen Meinung zu tun hatte.

Damit braucht man sich eigentlich nur selbst beim Wort zu nehmen: Was wir abbilden, sind nicht Personen an sich, sondern rekonstruierte Bil-

19 So die Web-Site des Projekts (https://de.dariah.eu/personendatenrepositorium); ähnlich: http://www.bbaw.de/forschung/personendaten-repositorium/ubersicht. 
der von Personen. Das mag haarspalterisch erscheinen, hat aber doch einige Konsequenzen. Eine Person existiert oder existiert nicht. Tertium non datur. Rekonstruktionen dagegen können überzeugend oder widerlegt sein, sie können dem Stand der Kenntnisse entsprechen oder auch nicht und es kommt vor, dass konkurrierende Rekonstruktionen nebeneinander bestehen, ohne dass man beim jetzigen Stand des Wissens eindeutig zwischen richtig und falsch entscheiden kann. Aber so funktioniert Forschung, und eine neue PIR würde Teil davon sein.

Daraus sind Folgerungen zu ziehen. Vor allem kann man ein solches Unternehmen nicht mit ein paar rasch angelernten Hilfskräften betreiben, die einfach Daten in den Computer eintippen. Für die Kernarbeit benötigt man Wissenschaftler, die mit Rekonstruktionen umgehen, diese beurteilen und - wo nötig - solche selbst erarbeiten können.

Ferner müssen die angebotenen wissenschaftlichen Meinungen dauerhaft auffindbar und zitierfähig bleiben. Wenn ein Benutzer die PIR kritisiert, muss also der betreffende Datensatz in der besprochenen Form weiter greifbar sein. Das steht etwas im Gegensatz zu dem Bestreben, den jeweils besten und aktuellsten Stand des Wissens anzubieten. Man wird also eine Versionierung einführen müssen - freilich man muss die archivierten älteren Bearbeitungsstände nicht in der Hauptansicht präsentieren. Technisch ist dies längst möglich, denn mit dem gleichen Problem sind viele digitale Publikationen konfrontiert. ${ }^{20}$ So kann man hier auf erprobte Werkzeuge zurückgreifen; man muss das Rad nicht neu erfinden.

Außerdem benötigt man ein Verfahren, um konkurrierende Rekonstruktionen abzubilden. In der Sache wäre das nichts Neues; auch in der gedruckten PIR wurden wichtige und wohlbegründete Gegenmeinungen stets angeführt. Wie dies technisch umzusetzen ist, bliebe im Detail noch auszuarbeiten.

Die Einträge als wissenschaftliche Meinungen zu verstehen, würde es schließlich auch erleichtern, sinnvolle Beziehungen zu anderen digitalen prosopographischen Werken herzustellen. Zu den Möglichkeiten der Verbindung zwischen verschiedenen Sammlungen hat eine Forschergruppe um Gabriel Bodard bereits eine Reihe von Erprobungen durchgeführt, ${ }^{21}$ doch es

20 Es genügt, auf die »Wikipedia» zu verweisen (https://de.wikipedia.org/wiki/ Hilfe:Versionen).

21 Das Projekt nannte sich SNAP:DRGN (https://snapdrgn.net/). Ziel war es, Standards für derartige Verknüpfungen zu entwickeln. Ein Nachfolgeantrag wurde vorerst nicht genehmigt. 
bleiben offene Fragen. Ein Beispiel: Im (auch online verfügbaren) `Lexicon of Greek Personal Namesı werden Zeugnisse einer einzigen Person zugeschrieben, wo die digitale Namensliste der PIR (und natürlich die gedruckte PIR) von der Existenz mehrerer Personen ausgeht. ${ }^{22}$ Wenn man nur die Kategorien `Person identisch Fällen kaum zu einer logisch sauberen Verknüpfung kommen (wie sie ein Datenbank-Programm voraussetzt). Wenn man akzeptiert, dass es um differierende Rekonstruktionen geht, täte man sich - zumindest logisch - wesentlich leichter. Denn dann kann es weitere Arten von Korrelationen geben, nämlich steilidentisch $`$ in verschiedenen Graden (wobei man jeweils spezifizieren müsste, was identisch ist und was nicht). Das impliziert auch, dass es von einem Datensatz aus Beziehungen zu mehreren Einträgen in einem anderen Sammelwerk geben kann (und dass auf ihn von mehreren anderen verwiesen wird), ohne dass man daran Anstoß nehmen müsste.

\section{Datenbank-Systeme}

Wenn man hinlänglich weiß, was man anstrebt, kann man sich dem technischen System zuwenden, das für die praktische Arbeit aufgebaut werden muss. Hier ist zunächst eines festzuhalten: Eine fertige Lösung, die eine digitale PIR einfach übernehmen könnte, existiert derzeit nicht. Die gängigen Datenbank-Programme liefern ohnehin jeweils nur das Werkzeug und den Unterbau, um eine Datensammlung erstellen und betreiben zu können. Hingegen müssen deren inhaltliche Ausgestaltung und die Strukturierung der Daten jeweils für den konkreten Anwendungsfall eigens erarbeitet und implementiert werden. Für manche vielgefragte Verwendungszwecke existieren fertige Einrichtungen - für prosopographische Aufgaben aber nicht. Auch wenn man auf Standardsoftware zurückgreift, würde man diese also in erheblichem Maße anpassen müssen. Umso genauer sollte man Umschau halten, welche Art von technischer Basis am besten zu den inhaltlichen Erfordernissen passt. ${ }^{23}$

22 Es gab zu dem Thema eine längere E-Mail-Korrespondenz zwischen Gabriel Bodard und dem Verfasser.

23 Auch das \Open Encyclopedia System` (http://www.open-encyclopedia-system. org bzw. http://www.cedis.fu-berlin.de/cedis/projekte/oes/index.html) hilft hier wenig weiter. Dabei geht es um kollaborativ erstellte Lexika in der Art der >Wikipedia`, wie ein bereits publiziertes Beispiel zeigt (die von der DFG geförderte International Encyclopedia of the First World Warı, http://www.1914-1918- 
Die heute noch immer vorherrschenden Datenbanken sind auf die Verwendung der "Structured Query Language» (SQL) hin ausgerichtet. Sie beruhen in ihrer Logik auf einem recht starren Grundschema von Tabellen mit genau definierten Inhalten; die Tabellenzeilen können jeweils durch Schlüsselelemente (eindeutige Identifikatoren) mit den Zeilen anderer Tabellen verknüpft werden. Dies alles setzt eindeutige und gleichartige Daten sowie eindeutige Relationen voraus. Optimal einzusetzen sind Datenbanken dieser Art, wo sehr große Mengen gleichartiger Daten sehr schnell auf immer wieder dieselben Fragen hin durchsucht werden müssen. ${ }^{24}$

Bei einer digitalen PIR müsste man hingegen mit einer deutlich anderen Grundlage zurechtkommen. Zum einen wäre die Menge der Daten vergleichsweise klein - jedenfalls im Verhältnis zu dem, was Datenbanken zu leisten vermögen. Zum anderen sind diese Daten recht vielgestaltig - jedenfalls gemessen an der Gleichförmigkeit eines modernen Telefon- oder Adressbuchs, für das man eine SQL-Datenbank gut einsetzen kann. Dort werden jeweils nur ausgewählte Angaben verarbeitet, die in allen Fällen eindeutig ermittelt werden können. Bei der PIR muss man sich hingegen mit dem begnügen, was mehr oder minder zufällig überliefert ist. Außerdem wird man ungern um der Gleichförmigkeit willen überlieferte Informationen weglassen, die für zukünftige Forschungsfragen relevant sein könnten. Ferner ist es erforderlich, überall (wie in der gedruckten PIR) jeweils die Quellenbelege und deren spezifische Art (kodiert) anzuführen. Öfters sind die Angaben nicht überliefert, sondern nur erschlossen, und in diesen Fällen sollte man wenigstens kurz die Argumente nennen. Schließlich sollte eine digitale PIR als Forschungsinstrument die Möglichkeit bieten, Fragen von sehr unterschiedlicher Art zu stellen. Andernfalls wäre ihre Nutzbarkeit ohne Zweifel stark eingeschränkt. Wollte man also eine digitale PIR mit einem SQL-System realisieren, müsste man wohl viele Kompromisse machen und die Daten zum Teil wohl auch gewaltsam in ein unflexibles Schema pressen.

So liegt es nahe, nach einer anderen Art von Technik zu suchen. Durchaus erfolgversprechend scheint es, ein Datenbank-System in Erwägung zu ziehen, das auf XML (`Extensible Markup Language ) beruht. Hier liegen eigentlich Texte zugrunde - allerdings Texte, die durch Auszeichnungsmerk-

online.net/). Diese Lösung ist viel zu unspezifisch für die Erfordernisse einer digitalen Prosopographie.

24 Auch die Epigraphik-Datenbank Clauss/Slaby (http://www.manfredclauss.de) beruht nach Kenntnis des Verfassers auf einer SQL-Datenbank. 
male strukturiert und durch standardisierte, nicht für den menschlichen Leser bestimmte Zusatzinformationen (»tags") angereichert sind und die deswegen auch maschinell verarbeitet werden können. Im Idealfall könnte man also eine Datenbank schaffen, die dem Benutzer zugleich einigermaßen lesbare Lexikon-Artikel bietet (und nicht nur Tabellen mit Einzelangaben). XML ist zudem vollständig dokumentiert, es ist lizenzfrei verfügbar, nicht an ein bestimmtes Programm gebunden und - nach allem, was man gegenwärtig wissen kann - das Dauerhafteste, das es derzeit in der Computerwelt gibt. HTML (die Sprache der Internet-Seiten) ist eigentlich nur eine Abart von XML, sodass es recht leicht fällt, XML-Daten ins Internet zu bringen. XML-Systeme wurden mit großem Erfolg für digitale online-Editionen verwandt (wie z. B. für die Inschriften von Aphrodisias von Charlotte Roueché) ${ }^{25}$ Aber inzwischen gibt es auch native XML-Datenbank-Programme, sogar kostenlos. ${ }^{26}$ Solche Systeme sind in mancher Hinsicht flexibler als SQLDatenbanken. Es würden auch keine Informationen verloren gehen, wenn die Daten irgendwann einmal exportiert werden müssen. Für die maschinengerechte Formulierung von - auch sehr komplexen - Suchanweisungen steht mit 'XQuery` eine Abfragesprache zur Verfügung, die sich in ihrer Leistungsfähigkeit durchaus mit SQL messen kann. Und wenn man sich für XML entscheidet, täte man sich schließlich verhältnismäßig leicht, die bisherige Materialsammlung der PIR in transformierter Form als Ausgangspunkt für das neue System nutzen.

XML-Datenbanken sind allerdings insgesamt langsamer als SQL-Datenbanken. Aber bei den Mengen, um die es hier geht, kann ein Mensch den Unterschied kaum wahrnehmen. Bei einem Test wurde die gesamte jetzige Materialsammlung der PIR - immerhin ca. 15.000 Einträge - mit der XML-Datenbank BaseX durchsucht: Es dauerte 0,09 Sekunden, und dies auf einem sehr alten, leistungsschwachen Rechner. Der Aspekt der Geschwindigkeit ist also kaum relevant.

Doch hat die schöne XML-Welt auch einige ernsthafte Nachteile. Die Struktur einer XML-Datei wird durch eine `Document Type Definition`(DTD) oder ein XML-Schema (XSD) definiert, die in einer eigenen Datei abgelegt werden. Solche Dateien kann man prinzipiell selbst schreiben, was jedoch aufwendig ist und Fachkenntnisse verlangt. Erheblich praktischer wäre es, wenn man ein fertiges und ausgereiftes Schema übernehmen kann. Für Texteditionen gibt es bereits solche Vorlagen, die sich inzwischen gut bewährt

25 http://insaph.kcl.ac.uk/ala2004/; http://insaph.kcl.ac.uk/iaph2007/.

26 Beispielsweise BaseX (http://basex.org/) und eXist (http://exist-db.org). 
haben (besonders die der »Text Encoding Initative«, TEI, und speziell für antike Inschriften das daraus weiterentwickelte EpiDoc) ${ }^{27}$ Aber für ein Prosopographie-Projekt gibt es nach meiner Kenntnis nichts dergleichen. Hier müsste man also noch Entwicklungsarbeit leisten.

Anders als bei konventionellen Datenbanken lässt sich die Haupt-Datei einer XML-Datenbank notfalls wie eine gewöhnliche Text-Datei öffnen. Aber sie ist für Nichtspezialisten schwer lesbar und noch weniger leicht zu bearbeiten. Man müsste also Werkzeuge schaffen, um Datensätze und Tags hinzuzufügen oder zu ändern, ohne `händisch` am XML-Quellcode arbeiten zu müssen. Denn nach einer Startphase sollten sich die Bearbeiter auf die Inhalte konzentrieren können und nicht viel über die Technik nachdenken müssen.

Erst recht müsste für die externen Benutzer, die dann über das Internet zugreifen, eine Oberfläche geschaffen werden, die unkompliziert zu handhaben ist. Ein XML-System eröffnet mehr als eine SQL-Datenbank die Möglichkeit, einen lesbaren Text anzubieten. Aber darüber hinaus müssen auch Abfragemöglichkeiten verfügbar gemacht werden, denn sonst würde ein Großteil des Potentials der neuen Publikationsform verschenkt. Dies könnte auf zwei Ebenen geschehen: einfach zu handhabende Standard-Recherchen für (mutmaßlich) häufig wiederkehrende Fragen und erweiterte Recherchen für speziellere Fragen, wofür dann dem Benutzer möglicherweise ein gewisser Lernaufwand zugemutet werden muss.

Alles in allem scheint es kein prinzipielles Hindernis zu geben, um eine digitale PIR auf einem XML-Datenbank-System zu implementieren. Doch sollten man sich darauf gefasst machen, etliches neu erfinden und entwickeln zu müssen, und möglicherweise wird man dabei auch einiges Lehrgeld zahlen müssen.

\section{Datensätze}

Zugleich mit den technischen Fragen muss überlegt werden, wie die zu verarbeitenden Daten strukturiert werden sollen. Einiges Prinzipielle dürfte für alle Arten von Datenbanken gelten, während die Einzelheiten dann auf das gewählte technischen System hin abgestimmt werden müssen.

So gilt es stets zu überlegen, welche Angaben logisch in einem Datensatz zu vereinen sind. Ferner muss man Klarheit gewinnen, zu welchen Fragen es

27 http://www.tei-c.org/index.xml; https://sourceforge.net/p/epidoc/wiki/Home/. 
immer nur eine einzige Antwort gibt und bei welchen jeweils mehrere vorkommen können - so hat ein Mensch nur einen Geburtstag, aber der Name kann sich durch Adoption ändern; auch kann jemand nacheinander mehrere Ehepartner und eine Vielzahl von Freunden haben. Generell gilt es in Datenbanksystemen zu vermeiden, dass die gleichen Daten an mehreren Stellen eingegeben (also wiederholt) werden - das ist eine notorische Fehlerquelle. Vielmehr sollten solche Daten nur an einer Stelle gepflegt werden; anderswo sollte man mit Verweisen auf diese Stelle statt mit Wiederholungen arbeiten. Entsprechend enthalten fast alle komplexeren Datenbanken intern mehrere, untereinander verbundene Datensammlungen. Dies ist auch für eine digitale PIR ins Augen zu fassen, denn sonst müsste man z. B. an mindestens 22 Stellen wiederholen, um welche Art von Zeugnis es sich beim Album Canusinum ${ }^{28}$ handelt und wo es publiziert ist, oder - wenn man auch Dekurionen aufnimmt - sogar an 147 Stellen. Es würde also neben der Sammlung für die Personen mindestens jeweils eine eigene Sammlung für die Testimonien und eine weitere für die Fachliteratur geben müssen, deren Einträge dann jeweils mit der entsprechenden Stellen bei den Personen zu verknüpfen sind. Bei den Testimonien wäre zwischen Inschriften, Papyri, Münzen und Notizen in der antiken Literatur zu unterscheiden und es müssten jeweils all die Angaben gemacht werden, die man für eine prosopographische Auswertung benötigt. Bei Inschriften würden demnach nicht nur die Publikationen genannt (und Links zu elektronischen Publikationen und online verfügbaren Fotos eingefügt), sondern auch der Typ des Monuments und sein Aufstellungsort registriert werden müssen.

Den Kern der Datenbank werden zweifellos die Datensätze zu den Personen ausmachen (oder genauer: zu den dokumentierten Rekonstruktionen). Hier bedarf es genauer Überlegung, denn für die Darstellung antiker Personen gibt es keine fest etablierten Standards. So muss eine digitale PIR ihren eigenen Weg finden. Dabei sollte vermieden werden, nur diejenigen Daten auszuwählen, die zur Beantwortung einiger weniger, vordefinierter Fragen taugen. ${ }^{29}$ Da die Informationen - wie ausgeführt - meist knapp, lückenhaft

28 CIL IX 338 = ILS 6121 - bekanntlich das inschriftlich überlieferte Verzeichnis der Mitglieder des Decurionenrats von Canusium in Süditalien aus dem Jahr 223 n. Chr., das auch die Namen ihrer zahlreichen hochmögenden Patrone enthält.

29 Man könnte einwenden, dass man nur etwas finden kann, wenn man weiß, wonach man sucht. Daran ist vieles richtig, aber dem menschlichen Gehirn genügt eine ungefähre Vorstellung. Wichtig wäre aber sicherzustellen, dass die Maschine erfassen und ausgeben kann, wonach der Mensch eigentlich sucht, 
und verschiedenartig sind, sollte man im Gegenteil möglichst viel registrieren und dabei ein erhebliches Maß an Variabilität zulassen. Ein Mindestmaß an Strukturierung ist aber unumgänglich.

Zunächst ist ein technisches Erfordernis zu beachten: Jeder Datensatz benötigt ein eindeutiges, nur einmal vorkommendes Kennzeichen, um ihn gezielt ansprechen zu können. Der Personenname kann das bei Römern natürlich nicht sein; denn oft trugen mehrere Personen genau den gleichen Namen. Auch sonst gibt es kein eindeutiges Merkmal, das in den Quellen bezeugt ist. Man wird also eine künstliche Referenznummer oder einen Code einführen müssen - und eine davon abgeleitete Referenz für die verschiedenen Versionen eines Datensatzes. Das ist auch wichtig für die Verknüpfungen mit anderen Projekten.

Manche Personen haben bereits einen Identifizierungscode in anderen großen Sammlungen, und diesen sollte man wohl ebenfalls registrieren. Wenn es beispielsweise für eine Person einen Eintrag in der >Gemeinsamen Normdatei (GND) der Bibliotheken gibt, sollte man darauf hinweisen. Beispielsweise existiert für Tacitus bereits ein solcher. ${ }^{30}$

Als eine Erweiterung hierzu kann man ansehen, was in neueren prosopographischen Arbeiten ohnehin Usus geworden ist: Konkordanzen zur Behandlung der Person in anderen Standardwerken. Der Gedanke erscheint grundsätzlich sinnvoll. Das allererste wäre die Nummer der gedruckten PIR, sofern vorhanden. Bei Frauen senatorischen Standes würde man aber sicher auch auf das Werk von Marie-Thérèse Raepsaet-Charlier ${ }^{31}$ hinweisen - und so fort. Als Dienstleistung für die Forschung könnte man auch die einschlägige Fachliteratur auflisten. Doch sollte man sich auf das Wesentliche beschränken statt den Benutzer mit Mengen an irrelevanten Hinweisen zu überschütten.

Nach diesen eher administrativen Angaben nun zu den Personen selbst: Hier sollte in jedem Fall der Name registriert werden - oder richtiger wie in der gedruckten PIR: die verschiedenen Namensformen mitsamt den Belegen. Man kann dann einen als den mutmaßlich korrekten herausheben.

und dass der Wunsch des Menschen richtig in maschinengerechte Befehle übersetzt wird. Zudem sollte man nicht von vornherein unterbinden, was man heute oft serendipidy nennt - glückliche Zufallsfunde, die fruchtbare weitere Forschungen anstoßen.

30 Für Tacitus (PIR ${ }^{2}$ C 1467) ist dies: idn=118620452 bzw. http://d-nb.info/gnd/ 118620452.

31 M.-Th. Raepsaet-Charlier: Prosopographie des femmes de l'ordre sénatorial (I. - II. siècles), 2 Bde., Louvain 1987. 
Lebensdaten sowie Geburts- und Todesort sind nur gelegentlich überliefert. So sollte man wenigstens alle Datumsangaben zu einzelnen Punkten im Lebenslauf notieren. Daraus kann man - per Hand oder mit Hilfe der Maschine - die ungefähre Lebenszeit ableiten.

Ein eigenes, gesondertes Verzeichnis aller inschriftlichen und papyrologischen Testimonien, in denen die Person erwähnt wird, benötigt man aber vielleicht nicht; man kann es sich - wo erforderlich - von der Maschine aus den verwendeten Quellenangaben extrahieren lassen.

Für die weiteren Angaben hat die gedruckte PIR bei den größeren Artikeln so etwas wie eine implizite Struktur entwickelt, an der man sich grob orientieren kann. Selbstverständlich ist der Rang zu notieren (als Senator, Ritter oder Dekurione oder als Frau oder Kind eines solchen), und wo Rangtitel bezeugt sind, auch diese. Selbstverständlich sind die öffentlichen Ämter und Funktionen einer Person zu erfassen, denn die Zugehörigkeit zum >Adel stand nach römischem Verständnis in unmittelbarem Zusammenhang damit. Wo es eine ein feste Abfolge von Ämtern gab (d.h. einen cursus honorum), muss diese ebenfalls abgebildet werden. Von zentraler Bedeutung sind es ferner, die Verwandten zu registrieren und auch die übrigen Personen, zu denen eine enge Beziehung bestand (wie Freunde, Patrone, Klienten etc.) - nicht nur, weil sich der Rang einer Frau über ihren Vater oder Ehemann definierte, die Vorfahren und Verwandte für das öffentliche Ansehen einer Person hoch bedeutsam waren und die engen Sozialbeziehungen zu den stärksten Kräften gehörten, die die Gesellschaft zusammenhielten. Es gehört darüber hinaus zu den klassischen Anwendungen der Prosopographie, die personalen Zusammenhänge zwischen den politisch handelnden Personen aufzudecken - was unter Umständen auch die Ereignisgeschichte sehr erhellen kann. ${ }^{32}$ Es dürfte allerdings genügen, den Namen, den Verwandtschaftsgrad oder die Art der Sozialbeziehung und die Quelle anzugeben und ansonsten auf den Datensatz zu verweisen, der für die entsprechende Personen angelegt wurde.

Schließlich sollten auch Ortsbeziehungen notiert werden. Wenn man Angehörige des ordo decurionum aufnimmt, muss ohnehin festgehalten werden, wo die Ämter ausgeübt wurden. Auch Ehrungen und Weihungen sind zu registrieren, ferner wo jemand Grundbesitz und Häuser hatte oder wo

32 Als gelungene Beispiele siehe R. Syme: The Roman Revolution, Oxford ${ }^{2} 1952$; W. Eck: An Emperor is Made. Senatorial Politics and Trajans Adoption by Nerva in 97, in: G. Clark / T. Rajak (Hgg.): Philosophy and Power in the GraecoRoman World. Essays in Honour of Miriam Griffin, Oxford, 2002, 211-226. 
er oder sie sich nachweislich länger aufhielt. In einigen Fällen kann man auch so etwas wie ein Itinerar von Personen rekonstruieren oder personale Netzwerke geographisch darstellen. Bei geographischen Angaben böte sich eine Verlinkung mit Atlas-Projekten (wie Pleiades) ${ }^{33}$ an.

Möglicherweise bräuchte man noch eine offene Rubrik, in der Besonderheiten notiert werden können - etwa dass jemand als Autor von literarischen Werken hervorgetreten ist, die noch erhalten sind, oder dass jemand ausgefallene Hobbys hatte.

So ergibt sich vielleicht ein Datensatz, der zum einen wie ein online-Lexikonartikel gelesen werden kann und Links bietet, die zu weitergehenden Informationen führen, und der zum anderen nach Art eines DatenbankEintrags durchsucht werden kann.

Ein technisch ebenso interessantes wie schwieriges Sonderproblem sind die Stemmata. In der gedruckten PIR wurden Verwandtschaftsbeziehungen durch Graphiken veranschaulicht, die freilich manuell erstellt waren. Prinzipiell ließen sich Stemmata auch maschinell generieren - soweit man eben Daten besitzt, wer von wem abstammte. ${ }^{34}$ Erst damit wären die Stemmata mit den digitalen Daten wirklich verknüpft. Aber mit einem solchen Vorhaben beträte man wohl technisches Neuland. Hinzu kommt, dass es über die Abstammung der hier zu bearbeitenden Personen oft nur gelehrte Konjekturen gibt - was zu berücksichtigen wäre. Andererseits könnte die Maschine möglicherweise Verbindungen auffinden, die der menschliche Stemma-Zeichner übersehen hat. Im Augenblick ist die Sache jedenfalls noch nicht spruchreif.

Auch sonst könnte man aus den Daten Graphiken generieren lassen, z. B. über Personenverflechtungen (wie es teilweise in der Netzwerk-Forschung getan wird). Wie viel dabei an zusätzlicher Erkenntnis zu gewinnen wäre, lässt sich aber derzeit nicht übersehen. ${ }^{35}$ Möglicherweise bleibt es bei der Illustration des ohnehin Bekannten.

33 https://pleiades.stoa.org/.

34 Dr. Klaus Prätor, der frühere Leiter der IT-Abteilung der BBAW, hat mich in diesem Zusammenhang auf Expertensysteme aufmerksam gemacht, die mit Baumdiagrammen arbeiten, und insbesondere auf die Programmiersprache PROLOG. Möglicherweise kann man also Hilfe von ganz unerwarteter Seite bekommen. Dies bliebe aber noch auszuloten.

35 Siehe http://historicalnetworkresearch.org/. - Der schlagende Nachweis eines effektiven Nutzens der Netzwerktheorie für die Alte Geschichte steht noch aus. 


\section{Sprache}

Die Sprachenfrage wurde bisher bewusst zurückgestellt, da sie - richtig betrachtet - eigentlich ein nachgeordnetes Problem sein dürfte. Bei einer reinen SQL-Datenbank wäre die Sprache kaum von Belang. Bei einem XMLSystem, das natürlichen Sprachen näher steht, ist die Frage nicht ganz zu umgehen.

Eines dürfte feststehen: Eine neue PIR wird man nicht mehr in Latein abfassen. Auch bei PIR ${ }^{2}$ war das bis zu einem gewissen Grad schon eine Antiquität. Für die Aufgabe von Latein gibt es verschiedene Gründe - schlechte und gute. Dass die Lateinkenntnisse zurückgehen, ist unübersehbar, und offenbar ist dies auch bei professionellen Altertumswissenschaftlern zu konstatieren, obwohl es eigentlich nicht sein dürfte. Darüber zu klagen hilft nicht; man wird sich nüchtern fragen müssen, ob es Sinn hätte, ein System aufzubauen, dass wissentlich an den Wünschen und Erwartungen der Benutzer vorbeigeht. Zudem gibt es sachimmanente Gründe, die gegen Latein sprechen. Bei PIR ${ }^{2}$ ist es zum Teil zu einer Vermischung von Zitat, Paraphrase und Kommentar gekommen, die nicht wünschbar ist. Wenn in einer anderen Sprache kommentiert wird, bleibt der Unterschied deutlicher.

Aber welche Sprache, wenn nicht Latein? Englisch ist zwar weit verbreitet, aber eigentlich schwer und nuancenreich; viele Nicht-Muttersprachler überschätzen ihre Englischkenntnisse deutlich. In den Altertumswissenschaften ist es zudem üblich, eine Mehrzahl moderner Sprachen zuzulassen (Englisch, Französisch, Deutsch, Italienisch und Spanisch). Das könnte man auch hier so handhaben; dann würde die Sprache letztlich vom jeweiligen Bearbeiter abhängen. Am Ende könnte man sogar mit einem System leben, das Einträge in unterschiedlichen Sprachen enthält. ${ }^{36}$

Jedoch sollten in einer digitalen PIR die Erläuterungen knapp und möglichst eindeutig gehalten werden. Längere, komplexe Erläuterungen sollte man besser in Zeitschriftenaufsätze auslagern und diese dann zitieren. Notfalls könnte man das System um ein Repositorium für Miszellen erweitern, in dem diese zitierbar hinterlegt werden. Wenn man so vorgeht, bliebe die Wahl der Sprache in der Tat ein zweitrangiges Problem.

36 Mehrsprachige Publikationen sind inzwischen keine Neuheit mehr, man vergleiche nur E. M. Steinby (Hg.): Lexicon Topographicum Urbis Romae, Bd. 16, Rom 1993-2000; Supplementum 1-7, Rom 2005-14. 


\section{Organisatorische Erfordernisse}

Technisch wäre eine digitale PIR also möglich, wenn auch unter nicht geringem Aufwand. Aber wie könnte man ein solches Vorhaben in der heutigen Wissenschaftslandschaft umsetzen? Hier ergeben sich erhebliche Schwierigkeiten. Bücher kann man Band für Band publizieren, aber mit einem Datenbank-System sollte man erst an die Öffentlichkeit treten, wenn es bereits gut als Arbeitsmittel nutzbar ist; nur dann wird es Anerkennung finden. Grob geschätzt sollten bereits um die $90 \%$ der geplanten Einträge verfügbar sein. Um in vernünftiger Zeit zu einem guten Ergebnis zu kommen, bräuchte man also in einer Startphase erhebliche Ressourcen, d.h. Stellen für IT-Fachleute und für ausgewiesene Wissenschaftler. Erst danach könnte man das Personal reduzieren und sich darauf beschränken, die anfallenden Aktualisierungen einzuarbeiten (und das System technisch aktuell zu halten). Wenn es zu lange dauert, bis man den take off point erreicht, wird man immer mehr Ressourcen in die inzwischen nötig werdende Überarbeitung der Datensätze stecken müssen. Es ist aber eher unwahrscheinlich, dass man für die Startphase - sagen wir zehn Stellen bekommt.

Vielleicht könnte man das eben angesprochene Problem teilweise entschärfen, wenn man sich für ein modulares Vorgehen entscheidet. Es wurde hier ein recht elaboriertes System skizziert, um die Zielrichtung zu verdeutlichen. Aber möglicherweise braucht man nicht alles sofort. In einer ersten Ausbauphase könnte man sich auf die wichtigsten Kerndaten zu den Personen beschränken - die allerdings zu definieren wären (es könnten der Name, die Verweise auf die relevanten Quellen und Fachpublikationen, die wichtigsten Ämter sowie die eindeutig bezeugten Personenverbindungen sein - und man könnte dies zunächst auf die Auswertung der gängigen Standardwerke gründen statt sofort alles erfassen zu wollen). Weiteres könnte man dann später hinzufügen. Manfred Clauss ist bei seiner EpigraphikDatenbank einen ähnlichen Weg gegangen, und er hatte Erfolg. ${ }^{37}$ Man sollte diese Lehre ernst nehmen.

Noch größere Schwierigkeiten zeichnen sich ab, wenn man an den für später erhofften Dauerbetrieb zu denken wagt. Um die Daten, die Programme und die Servermaschinen auf dem Stand zu halten, benötigt man fortlaufend Geld und Personal. In reiner Freiwilligenarbeit ist das nicht zu leisten, zumal für Arbeiten an einer digitalen PIR nicht einmal immaterielle Belohnungen in Gestalt von akademischer Reputation oder der Anre-

37 Siehe oben Anm. 24. 
chenbarkeit als Qualifikationsnachweis in Aussicht stehen. Das ist generell das Schicksal von Grundlagenprojekten. Man bräuchte also eine nachhaltige finanzielle Unterstützung. Aber die Wissenschaftsförderung ist gegenwärtig sehr stark projektorientiert und die Förderprogramme machen immer mehr inhaltliche Vorgaben. Zwar bekommt man in Deutschland unter Umständen Geld auch für längerfristige Projekte, aber für die Finanzierung von Daueraufgaben scheint es kaum ein geeignetes Format zu geben. Zugespitzt könnte man sagen, dass man jetzt zwar die Techniken des 21. Jahrhunderts besitzt, aber sie mit Organisationsformen des 19. zu betreiben versucht. Doch an den Strukturen wird sich so schnell nichts ändern. Man wird also damit rechnen müssen, dass auch eine digitale PIR irgendwann für abgeschlossen erklärt wird und Einträge dann nach und nach veralten. Ganz vergeblich wäre die Mühe aber vielleicht trotzdem nicht. 
